\title{
Calculating Life Years from Transplant (LYFT): Methods for Kidney and Kidney-Pancreas Candidates
}

\author{
R. A. Wolfe ${ }^{a, *}$, K. P. McCullough ${ }^{a}$, \\ D. E. Schaubel ${ }^{\text {, }}$, J. D. Kalbfleisch ${ }^{b}$, S. Murray ${ }^{\text {, }}$ \\ M. D. Stegallc and A. B. Leichtman ${ }^{b}$ \\ a Scientific Registry of Transplant Recipients, Arbor \\ Research Collaborative for Health, Ann Arbor, MI \\ b Scientific Registry of Transplant Recipients, University of \\ Michigan, Ann Arbor, MI \\ 'Department of Surgery, Mayo Clinic, Rochester, MN \\ * Corresponding author: Robert A. Wolfe, \\ robert.wolfe@arborresearch.org
}

The Organ Procurement and Transplantation Network (OPTN) Kidney Committee is considering a proposal for a new deceased donor kidney allocation system. Among the components under consideration is a strategy to rank candidates in part by the estimated incremental years of life that are expected to be achieved with a transplant from a specific available deceased donor, computed as the difference in expected median lifespan with that transplant compared with remaining on dialysis. This concept has been termed life years from transplant or LYFT. Median lifespans could be calculated, based on objective medical criteria, for each candidate when a deceased donor kidney becomes available, based on Cox regression models using current candidate and donor medical information. The distribution of the calculated LYFT scores for an average nonexpanded criteria donor kidney is similar across candidate sex, race/ethnicity, insurance status and, with the exception of diabetes, diagnosis. LYFT scores tend to be higher for younger candidates and lower for diabetics receiving a kidney-alone rather than a simultaneous kidney-pancreas transplant. Prioritizing candidates with higher LYFT scores for each available kidney could substantially increase total years of life among both transplant candidates and recipients. LYFT is also a powerful metric for assessing trends in allocation outcomes and for comparing alternative allocation systems.

Key words: Cox regression, deceased donor kidneys, diabetes mellitus, kidney allocation, kidney transplant, life expectancy, OPTN, simultaneous kidney-pancreas transplant, SRTR, survival, waiting list, waiting list mortality

\section{Introduction}

In the United States, under the current Organ Procurement and Transplantation Network (OPTN) allocation system, de- ceased donor kidneys are primarily allocated through a combination of waiting time and human leukocyte antigen (HLA) matching. Additional elements address sensitized candidates, children, prior living donors, a payback system for shared kidneys and priority for candidates local to the deceased donor. Nationally, over the past decade, there has been a decline in both average posttransplant lifetimes and in the life-years gained through transplantation with standard criteria donor (SCD) kidneys (1).

In 2003, the OPTN Board of Directors charged the OPTN Kidney Committee (OPTNKC) with undertaking a '360 degree review' of the current kidney allocation system. During this process, the OPTNKC looked to the OPTN Final Rule (Federal Register, 20 October 1999, section 121.8) for guidance. The Final Rule requires that deceased donor organs should be allocated using objective medical criteria, de-emphasizing the role of waiting time, in order to 'achieve the best use of donated organs'. Among the allocation concepts developed collaboratively by the Scientific Registry of Transplant Recipients (SRTR) and the OPTNKC to comply with the requirements of the Final Rule, is life years from transplant (LYFT), based on earlier work by Wolfe et al. (2) LYFT is defined as the difference in expected median survival for a candidate with a kidney transplant from a specific donor and the expected median survival for that candidate without any transplant at all. These expected lifetimes with and without a kidney transplant are calculated based on the medical and demographic characteristics of each candidate. Survival with a kidney transplant incorporates characteristics of the donor kidney as well.

This article summarizes the methodologies proposed by the SRTR and under consideration by the OPTN to calculate LYFT scores for kidney and kidney-pancreas transplant candidates. The next section features a general overview of the methods underlying LYFT calculations, and subsequent sections elaborate on specific issues related to the LYFT models. Development of a practical kidney allocation system involves a balance among multiple and sometimes conflicting objectives. The OPTNKC is involved in a continuing multi-year examination of various ways to balance those objectives, including the potential incorporation of LYFT as a component in allocation.

\section{Overview of Methods}

Cox regression models, a proportional hazards model widely employed to analyze censored survival data (3), were used to estimate death rates and 
corresponding survival curves for candidates (yielding survival curve estimates without transplant) and recipients (yielding survival curve estimates with transplant). The survival curves were then used to estimate expected lifetimes. As described in the section below, 'Evaluating Proportional Hazards', stratification was employed to account for nonproportional baseline death rates among diabetics listed for or receiving kidney-alone transplants, diabetics listed for or receiving simultaneous kidney-pancreas (SPK) transplants and nondiabetics. Interaction terms, i.e. terms in the regression models that allow effects of covariates to differ between groups of patients, were used where statistically and clinically indicated. As will be discussed, separate models were built for short- and long-term survival to ensure that the assumptions of the model, especially the proportional hazards assumption, were satisfied. The proportional hazards model specifies that the covariates used in the regression model have a multiplicative effect on the death rate throughout the follow-up period.

Candidates and transplant recipients from 1987 to 2006 were followed for survival through 2006, with an adjustment for the year of start of followup. Candidate survival without a transplant was censored at the time of transplant or end of study (2006). Recipient survival with a transplant was censored at the time of repeat transplantation or end of study (2006). The graft lifetime estimates were based on graft failure, including death, reported graft failure and return to chronic dialysis, with censoring at end of study. The resulting survival curves were adjusted to account for the time period from which the data were obtained and were used to estimate the median expected patient survival with and without a transplant and the median expected graft survival. In some cases, the median survival was longer than 15 years (the maximum time at which the survival curve was estimated). In those circumstances, extrapolated death rates (described in the section below, 'Long-Term Survival and Extrapolation') were used to estimate median survival.

\section{Calculation of LYFT}

The LYFT score for each candidate is calculated using the estimated median survival times (measured in years) without a transplant, based on candidate characteristics, and with a transplant for each recipient (with and without a functioning graft), based on recipient and donor characteristics. To account for differences in the quality of life with a functioning graft compared with life on dialysis, each year on dialysis is adjusted by a factor of 0.8 , based on a synthesis of assessments in the published literature $(4,5)$, while each year with a functioning graft is given a value of 1.0. The resulting formula used to calculate LYFT is shown in Figure 1.

\section{Measuring Typical Lifetimes}

Several options were considered to estimate the typical lifetimes expected for a group of candidates with a particular set of characteristics. These options included 'expected lifetime' (area under the survival curve), 'truncated expected lifetime' (area under the survival curve limited to a specified interval, such as up to 10 years) and the 'median lifetime' often referred to as the half-life (i.e. the time at which half of the population has died). Each method provides a measure of the typical lifespan of patients or grafts

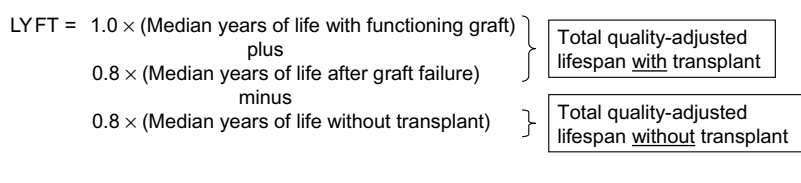

Source: SRTR, 2007.

Figure 1: Formula for LYFT.

bearing the specific characteristics of the candidate, recipient or graft for whom the lifespan is being estimated.

Typical lifetimes vary substantially among candidates and recipients. They can be as short as 1.4 years on dialysis alone and 4.2 years posttransplant for older or less healthy patients. For other groups, especially younger, healthier patients, more than $50 \%$ of patients were still alive 15 years posttransplant.

One of the challenges was to identify an appropriate metric of expected lifetime that would yield accurate and meaningful estimates at the extremes of age and health. It is important to recognize, since the LYFT calculation is the difference between expected posttransplant and waiting list survivals, that all else being equal, LYFT scores will be highest not only for candidates with relatively longer expected posttransplant survival but also for candidates with relatively shorter waiting list survival (medical urgency). Calculation of LYFT scores among subpopulations of candidates is elaborated below in the section, 'LYFT Scores versus Candidate Demographics'.

Life years as calculated from the area under the survival curve for a population was initially considered for calculating LYFT. Area under the survival curve estimates the average lifetime of that population. However, the area under the survival curve could not be calculated directly with available data for those populations for which a substantial fraction were still alive at the end of follow-up (15 years). Furthermore, the area under the survival curve, beyond the end of follow-up, cannot be estimated using standard Cox regression techniques. Therefore, alternative methods for estimating lifetimes were investigated.

The OPTNKC also considered calculations of lifetimes truncated at a fixed number of years, starting with 10 years, based on the area under the survival curve up to the prescribed number of years. Although this area can be calculated with the available data, the resulting metrics exclude from the survival calculation a portion of the LYFT that many, especially younger and healthier, candidates could expect to achieve. Potentially short-lived recipients tend to gain their LYFT soon after transplant (within 5 to 10 years), while longer-lived recipients are more likely to realize their LYFT several years later (15 to 25 years after transplant). Thus, truncation even at 10 or 20 years would arbitrarily exclude from survival calculations much of the LYFT that 


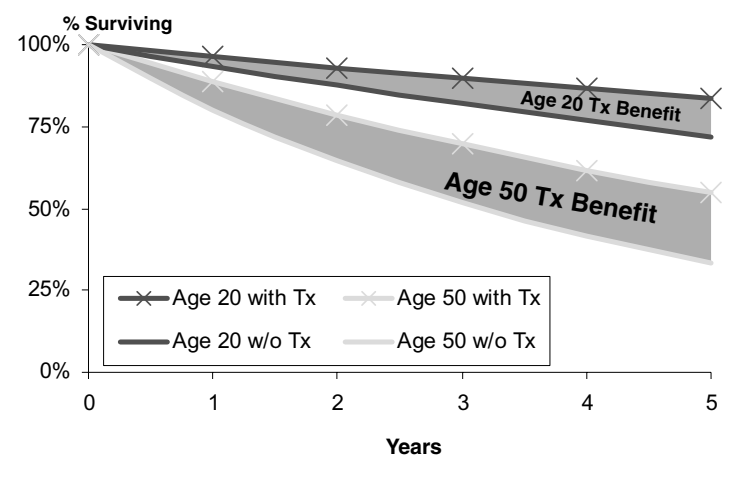

Source: SRTR, 2007

Figure 2: Hypothetical 5-year survival with and without transplant (Tx) for age 20 versus age 50 years.

is attained by long-lived candidates. This concept is illustrated in Figure 2 and Figure 3. In Figure 2, measurement of survival is truncated at 5 years for a hypothetical population of patients; the difference between the area under the resultant recipient and candidate survival curves is larger for patients transplanted at the age of 50 years than it is for those transplanted at 20 years. In Figure 3, greater than 30 years of posttransplant survival is hypothetically captured. In contrast to Figure 2, the difference between the area under the resultant recipient and candidate survival curves is now larger for 20-year-old patients than it is for those 50 years old. In addition, the OPTNKC is interested in minimizing the transplantation of kidneys with the potential for long posttransplant survival into recipients at high risk for early death with a functioning transplant. Thus, it was important to use a metric that was appropriate for longer-lived candidates.

In contrast, the median lifetime can be estimated for the majority of candidates without projection of the survival curve beyond 15 years. The median lifetime was less than 15 years and could thus be estimated directly from existing data for $99 \%$ of lifetimes without transplant and for $72 \%$ of posttransplant lifetimes with an average nonexpanded

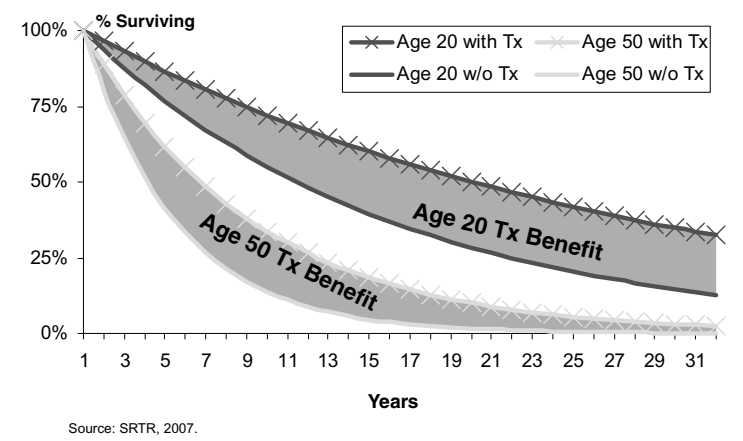

Figure 3: Hypothetical 32-year survival with and without transplant $(T x)$ for age 20 versus age 50 years. criteria donor (non-ECD) kidney. (Note: By the OPTN definition, ECD kidneys are defined as having a risk of graft failure $\geq 1.7$ times that of ideal donors. ECDs include any donor $\geq 60$ years and donors $50-59$ years with at least two of the following: terminal creatinine $>1.5 \mathrm{mg} / \mathrm{dL}$, history of hypertension, or death by cerebrovascular accident.) For the remainder of candidates and recipients, extrapolation methods were used to estimate the median lifetime (see 'Long-Term Survival and Extrapolation' below). The median estimated lifetimes were much more consistent for longer (over 15 years) lifetimes with alternative extrapolation methods than were those calculated using the area under the survival curve.

\section{Modeling}

Separate models were estimated for short-term (years 0 to $<4$ ) and long-term (years 4-15) survival with transplant, survival without transplant and graft survival, for a total of six models in all. The short-term models predicted survival out to 4 years, while the long-term models estimated survival to 15 years, conditional on surviving the first 4 years. The 4 year cut point was chosen because it best fit the shapes of the survival curves and because it is well beyond the period of elevated death rates that persisted for longer than 1 year following transplant for some patient groups. The same 4year cutoff also allowed the waiting list and posttransplant survival models to accommodate covariates with potentially nonproportional associations with short-term versus long-term death rates.

Survival without transplant was estimated based on death rates among patients who had been listed for transplantation. The data used to estimate survival without a transplant includes pretransplant data for those candidates who received a transplant, data for candidates who were waitlisted but not yet transplanted, data up until the time of death for candidates who died on the waiting list and follow-up data for candidates who were removed from the waiting list for reasons other than death or transplantation. Similarly, survival with transplant includes survival time after graft failure, censored at retransplantation. Graft survival was calculated as the time to death or graft failure, whichever occurred sooner. Lifespan after graft failure was calculated as the difference between lifespan following transplant and the lifespan of the functioning graft. Covariate selection was carried out separately for each of the six models. Short-term and long-term survival models were not required to contain the same variables, as described in the section below, 'Covariates Used to Estimate Short-Term and Long-Term Survival'.

\section{Data}

\section{Descriptive statistics}

Calculation of LYFT depends on models used to estimate survival both with and without a kidney transplant. Survival 
Table 1: Sample size

\begin{tabular}{cll}
\hline Count in sample & $0-<4$-year period & \multicolumn{1}{c}{ 4-15-year period } \\
\hline Without transplant & 118090 candidates & 26 335 candidates \\
model data & 21030 deaths & 7498 deaths \\
& 50593 transplanted & 5287 transplanted \\
With transplant & 131713 recipients & 83738 recipients \\
model data & 17341 deaths & 22933 deaths \\
& 33 292 graft failures & 28 121 graft failures \\
& 3507 retransplants & 6536 retransplants \\
\hline
\end{tabular}

without a kidney transplant is estimated using past waiting list data; survival with a kidney transplant is estimated using past data on kidney recipients.

Models used to estimate survival without a transplant are based on candidates active on the waiting list on 1 January of each of 1988, 1990, 1994, 1998 and 2002. These samples were aggregated to include both long-term survival (from older cohorts) and recent survival (from recent cohorts). Only adult candidates for deceased donor kidneys (even if they eventually received a living donor kidney) and recipients of deceased donor kidneys were included. Recipient survival was censored at the time of transplant for those candidates who received a subsequent kidney, but this retransplantation, if preemptive, counted as a graft failure in graft survival models.

Models of both patient and graft survival after transplant were based on all non-ECD kidney and SPK transplants from 1987 to 2006. Simultaneous kidney with extra-renal organ transplants, with the exception of SPK transplants, were excluded.

The resulting sample sizes for the different models and periods are shown in Table 1. Table 2 displays descriptive statistics of the data used to build the models for estimating the median waiting list and posttransplant survival times, and, as an example, for adult candidates active on a specific sample offer date (January 1, 2004). Using the cross section of active candidates on a given date provided general information on the demographics of typical candidates who might be active at the time of an organ offer. This cross section is used below in the section, 'LYFT Scores Versus Candidate Demographics', to illustrate differences in LYFT scores among an average group of candidates who might be available for transplant on a given date.

\section{Variables used or investigated for use in the LYFT calculation}

Effects in LYFT models. Table 3 contains hazard ratios from the Cox regression models used in the LYFT calculation for the variables employed in each survival estimate. Table 4 shows variables investigated for use but later excluded, along with the reasons for exclusion. These vari- ables were excluded if they were not predictive, not objective or not clinically relevant. Each hazard ratio indicates the relative change in risk associated with either a 1-unit increase in the covariate (for continuous covariates) or the change in risk for the group of candidates identified by the covariate relative to a reference group (for categorical covariates). Depending on the outcome used in the model, hazard ratios greater than 1 indicate increasing risk of either death or graft failure and hazard ratios less than 1 indicate decreasing risk. In both tables, each column represents a separate model, and cells within each column are left blank for variables not used for a particular model. In Table 3, the variables with shaded cells were not used in the 4- to 15-year models. The shaded cells indicate the hazard ratios that would have been obtained had each variable group been included one at a time along with the variables actually used.

There are separate models for the three types of survival.

1. WL (Waiting List): Candidate survival without transplant is calculated from a given calendar date, rather than the individual listing dates. The cross-sectional nature of the sample selection allows the survival patterns used in the LYFT calculation to mimic those seen in the prevalent waiting list population upon which allocation is based and not the incident waiting list, which contributes to but does not replicate the candidate list at any particular moment in time (6).

2. PT (Posttransplant): Recipient survival with a transplant. This includes all postoperative mortality, but survival time is censored on retransplant.

3. GS (Graft Survival): Graft survival after a transplant. For kidney-pancreas recipients, this is the survival of the kidney, not the pancreas graft.

Short- and long-term survival for each model was estimated separately. Short-term survival during the 0 to 4 -year period after a given calendar date, mimicking the survival of nonrecipients after an offer (WL) or of recipients after transplant (PT or GS), is indicated by ' 0 to $<4$ ' in the column header of Tables 3 and 4 , and long-term survival is indicated by '4-15'. Hazard ratios in bold italics show that the variable was significant $(p<0.05)$ in the specific model listed in the column header.

\section{Model Diagnostics}

In this section, we describe the methods used to evaluate the survival models upon which the LYFT calculations are based, beginning with a summary measure of a model's predictive capacity and followed by an assessment of its underlying assumptions. 
LYFT Methods

Table 2: Mean (SD) of variables used in LYFT calculations

\begin{tabular}{|c|c|c|c|}
\hline Variable mean or proportion shown (SD) & $\begin{array}{c}\text { Waiting list } \\
(1 / 1 / 04 \text { sample) }\end{array}$ & $\begin{array}{l}\text { Waiting list } \\
\text { (model data) }\end{array}$ & $\begin{array}{c}\text { Transplant } \\
\text { (model data) }\end{array}$ \\
\hline Kidney-alone (KI) diabetic (DM) & $0.31(0.46)$ & $0.26(0.44)$ & $0.22(0.41)$ \\
\hline Kidney-pancreas (KP) & $0.04(0.19)$ & $0.03(0.17)$ & $0.09(0.29)$ \\
\hline Candidate age at offer or transplant (years) & $49.63(12.73)$ & $46.56(12.66)$ & $45.93(12.48)$ \\
\hline Candidate body mass index (BMI) $\left(\mathrm{kg} / \mathrm{m}^{2}\right)$ & $26.95(5.67)$ & $25.91(5.46)$ & $25.78(5.15)$ \\
\hline Candidate BMI missing & $0.36(0.48)$ & $0.29(0.45)$ & $0.31(0.46)$ \\
\hline Candidate diagnosis: other/missing & $0.17(0.37)$ & $0.32(0.47)$ & $0.36(0.48)$ \\
\hline Candidate diagnosis: polycystic & $0.06(0.24)$ & $0.05(0.22)$ & $0.05(0.23)$ \\
\hline Candidate diagnosis: hypertension & $0.20(0.4)$ & $0.16(0.36)$ & $0.12(0.32)$ \\
\hline Candidate diagnosis: glomerular & $0.22(0.41)$ & $0.19(0.39)$ & $0.16(0.36)$ \\
\hline Candidate previous transplant (any) & $0.23(0.42)$ & $0.24(0.42)$ & $0.18(0.39)$ \\
\hline Candidate peak panel reactive antibody (PRA) $<10$ & $0.56(0.5)$ & $0.37(0.48)$ & $0.62(0.49)$ \\
\hline Candidate peak PRA 10-79 & $0.23(0.42)$ & $0.17(0.38)$ & $0.26(0.44)$ \\
\hline Candidate peak PRA 80+ & $0.15(0.36)$ & $0.1(0.3)$ & $0.11(0.31)$ \\
\hline Candidate peak PRA missing & $0.05(0.23)$ & $0.35(0.48)$ & $0.02(0.13)$ \\
\hline Year of offer or transplant & $2004(0)$ & $1997(4)$ & $1997(6)$ \\
\hline Candidate total albumin (g/dL) & $3.83(0.62)$ & $3.83(0.72)$ & $3.84(0.66)$ \\
\hline Candidate albumin missing & $0.41(0.49)$ & $0.82(0.39)$ & $0.77(0.42)$ \\
\hline Years since start of dialysis & $4.04(3.97)$ & $3.81(3.92)$ & $3.39(3.41)$ \\
\hline Candidate had not started dialysis as of offer & $0.07(0.26)$ & $0.07(0.25)$ & $0.07(0.25)$ \\
\hline 0 ABDR HLA mismatch (MM) & & & $0.13(0.33)$ \\
\hline O A HLA MM & & & $0.22(0.41)$ \\
\hline 1 A HLA MM & & & $0.38(0.49)$ \\
\hline 0 B HLA MM & & & $0.20(0.40)$ \\
\hline 1 B HLA MM & & & $0.35(0.48)$ \\
\hline O DR HLA MM & & & $0.30(0.46)$ \\
\hline 1 DR HLA MM & & & $0.44(0.50)$ \\
\hline Shared organ & & & $0.29(0.45)$ \\
\hline Donation after cardiac death & & & $0.03(0.16)$ \\
\hline Donor age (continuous years) & & & $32.4(13.48)$ \\
\hline Donor cytomegalovirus negative & & & $0.41(0.49)$ \\
\hline Donor hypertension & & & $0.08(0.28)$ \\
\hline Donor weight $(\mathrm{kg})$ & & & $74.98(19.74)$ \\
\hline Donor weight missing & & & $0.10(0.30)$ \\
\hline Donor cause of death: anoxia & & & $0.10(0.29)$ \\
\hline Donor cause of death: cerebrovascular accident & & & $0.31(0.46)$ \\
\hline Donor cause of death: central nervous system tumor & & & $0.01(0.09)$ \\
\hline Donor cause of death: other/unknown & & & $0.11(0.31)$ \\
\hline Donor cause of death: head trauma & & & $0.47(0.50)$ \\
\hline Candidate death observed during follow-up without transplant & $0.14(0.35)$ & $0.24(0.43)$ & \\
\hline Candidate duration of follow-up without transplant & $1.76(1.02)$ & $2.46(2.51)$ & \\
\hline Recipient death observed during follow-up after transplant & & & $0.29(0.45)$ \\
\hline Recipient duration of follow-up after transplant & & & $5.99(4.63)$ \\
\hline Recipient graft failure observed during follow-up & & & $0.44(0.50)$ \\
\hline Recipient duration of follow-up after transplant & & & $5.14(4.45)$ \\
\hline
\end{tabular}

\section{Predictive capability of the models}

The index of concordance (IOC) is an overall measure of goodness-of-fit that gauges a model's ability to successfully rank patient survival. The IOC is computed as the proportion of patient pairs for which the model correctly predicts ordering of the survival times. The IOC ranges from 0.5 to 1.0 , with 0.5 indicating that the model is unable to rank candidates and with 1.0 indicating that the model ranks candidates perfectly according to their survival. The IOC was calculated by using a random half of the available data to create the model and a random subsample of the other half of the available data to test the model's predictive capability. The IOC is provided for each model component in the first row of Table 3.

\section{Evaluating proportional hazards}

Based on statistical diagnostic tests, we identified factors that appeared to have nonproportional hazards effects. The following discussion lists the covariates for which the proportional hazards assumption did not hold and explains the procedures used to account for this nonproportionality. 
Table 3: Hazard ratios of variables used in LYFT calculation

\begin{tabular}{|c|c|c|c|c|c|c|}
\hline Factor & $\begin{array}{c}W L \\
0-<4\end{array}$ & $\begin{array}{c}\text { WL } \\
4-15\end{array}$ & $\begin{array}{c}\text { PT } \\
0-<4\end{array}$ & $\begin{array}{c}\text { PT } \\
4-15\end{array}$ & $\begin{array}{c}\text { GS } \\
0-<4\end{array}$ & $\begin{array}{c}G S \\
4-15\end{array}$ \\
\hline Overall IOC for model as used in LYFT' & 0.66 & 0.60 & 0.67 & 0.68 & 0.59 & 0.57 \\
\hline Candidate age at offer or transplant (per 10 years) & 1.344 & 1.384 & 1.411 & 1.466 & 1.010 & 1.116 \\
\hline $\begin{array}{l}\text { Candidate for or recipient of kidney alone and diabetic (KI } \\
\text { DM) }\end{array}$ & 1.975 & 1.764 & & & & \\
\hline $\begin{array}{l}\text { Candidate for or recipient of simultaneous } \\
\text { kidney-pancreas (KP DM) }\end{array}$ & 2.672 & 2.138 & & & & \\
\hline $\begin{array}{l}\text { Candidate for or recipient of } \mathrm{KI} \text { alone and non-diabetic (KI } \\
\text { non-DM) }\end{array}$ & (ref) & (ref) & (ref) & (ref) & (ref) & (ref) \\
\hline Candidate was not on dialysis by sample date & 1.466 & 0.848 & 1.414 & 1.210 & 1.312 & 1.157 \\
\hline $\begin{array}{l}\text { Candidate had not developed full end-stage renal disease } \\
\text { (ESRD) by sample date interaction with KI DM }\end{array}$ & 0.328 & 0.748 & 0.599 & 0.780 & 0.818 & 0.898 \\
\hline $\begin{array}{l}\text { Candidate had not developed full ESRD by sample date } \\
\text { interaction with KP DM }\end{array}$ & 0.710 & 0.830 & 0.796 & 0.913 & 0.851 & 0.936 \\
\hline Candidate body mass index (BMI) $\left(\mathrm{per} \mathrm{kg} / \mathrm{m}^{2}\right)$ & 0.966 & 1.018 & 0.933 & 0.960 & 0.966 & 0.982 \\
\hline Candidate BMI missing & 0.503 & 1.260 & 0.270 & 0.473 & 0.552 & 0.712 \\
\hline Candidate BMI change in slope $\left(\right.$ per $\left.\mathrm{kg} / \mathrm{m}^{2}>20\right)$ & 1.028 & 0.982 & 1.081 & 1.055 & 1.053 & 1.032 \\
\hline Candidate diagnosis: polycystic & 0.705 & 0.846 & 0.698 & 0.653 & 0.686 & 0.623 \\
\hline Candidate previous transplant (any) & 1.224 & 1.130 & 1.458 & 1.352 & 1.321 & 1.252 \\
\hline Peak panel reactive antibody (PRA) 10-79 (ref $=<10)$ & 0.971 & 0.979 & 0.999 & 1.010 & 1.049 & 1.031 \\
\hline Peak PRA $80+($ ref $=<10)$ & 1.003 & 1.018 & 1.160 & 1.135 & 1.299 & 1.095 \\
\hline Peak PRA missing (ref $=<10)$ & 1.043 & 1.143 & 1.080 & 0.940 & 1.081 & 1.046 \\
\hline Peak PRA $<10$ (reference) & (ref) & (ref) & (ref) & (ref) & (ref) & (ref) \\
\hline Year of offer or transplant (minus 1998) & 0.983 & 1.009 & 0.949 & 0.949 & 0.948 & 0.967 \\
\hline Candidate albumin (per g/dL) & 0.624 & 0.66 & 0.787 & 0.895 & 0.772 & 0.815 \\
\hline Candidate albumin change in slope (per g/dL >3.5) & 1.422 & 1.326 & 1.146 & 0.983 & 1.245 & 1.174 \\
\hline Candidate albumin missing & 0.220 & 0.272 & 0.416 & 0.638 & 0.396 & 0.465 \\
\hline 0 ABDR HLA mismatch (MM) & & & 0.915 & 0.907 & 0.747 & 0.87 \\
\hline 0 DR HLA MM & & & 0.873 & 0.926 & 0.853 & 0.916 \\
\hline 1 DR HLA MM & & & 0.943 & 0.983 & 0.928 & 0.962 \\
\hline $\begin{array}{l}\text { Shared organ (i.e. donor organ procurement organization } \\
\text { (OPO) } \neq \text { recipient OPO) }\end{array}$ & & & 1.068 & 1.035 & 1.087 & 1.035 \\
\hline Donation after cardiac death & & & 0.873 & 0.745 & 0.942 & 0.819 \\
\hline Donor age (per year) & & & 0.977 & 1.003 & 0.978 & 0.999 \\
\hline Donor age change in slope (per year $>18$ ) & & & 1.033 & 1.004 & 1.035 & 1.011 \\
\hline Donor cause of death: anoxia (ref = head trauma) & & & 1.028 & 1.024 & 1.034 & 1.021 \\
\hline $\begin{array}{l}\text { Donor cause of death: cerebrovascular accident (ref }= \\
\text { head trauma) }\end{array}$ & & & 1.137 & 1.101 & 1.153 & 1.07 \\
\hline $\begin{array}{l}\text { Donor cause of death: central nervous system tumor (ref } \\
=\text { head trauma) }\end{array}$ & & & 0.969 & 0.851 & 0.949 & 0.858 \\
\hline Donor cause of death: other/unknown (ref = head trauma) & & & 1.241 & 1.112 & 1.23 & 1.098 \\
\hline Donor cause of death: head trauma (reference) & & & (ref) & (ref) & (ref) & (ref) \\
\hline Donor cytomegalovirus negative & & & 0.952 & 0.975 & 0.949 & 0.964 \\
\hline Donor hypertension & & & 0.969 & 0.952 & 1.014 & 1.054 \\
\hline Donor weight in $\mathrm{kg}$ (per 1 unit increase in log of weight) & & & 0.876 & 0.967 & 0.814 & 1.035 \\
\hline Donor weight missing & & & 0.901 & 1.019 & 0.662 & 1.253 \\
\hline $\begin{array}{l}\text { Years since dialysis start (per } 1 \text { unit increase in log of } \\
\text { years) }\end{array}$ & 1.400 & 1.103 & 1.291 & 1.240 & 1.178 & 1.180 \\
\hline Candidate age interaction with KI DM & 0.974 & 0.984 & 0.984 & 0.983 & 1.007 & 1.004 \\
\hline Candidate age interaction with KP DM & 1.010 & 0.985 & 0.996 & 0.993 & 0.990 & 0.974 \\
\hline Candidate albumin interaction with KP DM & 1.005 & 1.013 & 0.978 & 0.932 & 1.009 & 0.956 \\
\hline Candidate BMI interaction with KP DM & 1.001 & 1.000 & 0.996 & 0.996 & 0.996 & 0.997 \\
\hline PRA 10+ interaction with KP DM & 0.780 & 1.151 & 1.005 & 1.063 & 0.960 & 1.098 \\
\hline Candidate previous transplant interaction with KP DM & 0.985 & 0.755 & 0.836 & 0.803 & 0.886 & 0.828 \\
\hline
\end{tabular}

Bold italics indicate $P<0.05$. Shading indicates variables not used in the model shown in the column header.

${ }^{1}$ Note: $I O C=$ index of concordance; $\mathrm{WL}=$ survival without transplant; PT = survival with transplant; GS = graft survival. The shaded cells display variables that were not included in the 4- to 15-year models. The hazard ratios in the shaded cells are those that would have been obtained had each variable group been included one at a time along with the variables actually used in the 4- to 15-year models.

${ }^{2}$ Posttransplant models are stratified by diabetes status and kidney versus kidney-pancreas transplant in the LYFT calculation due to the nonproportional hazard nature of the relationships of these variables with mortality. Thus hazard ratios for these factors are not constant over time. 
LYFT Methods

Table 4: Additional variables considered for LYFT calculation: hazard ratio (index of concordance [IOC])

\begin{tabular}{|c|c|c|c|c|c|c|c|}
\hline Factor & Reason not used & $\begin{array}{c}W L \\
0-<4\end{array}$ & $\begin{array}{l}\text { WL } \\
4-15\end{array}$ & $\begin{array}{c}\text { PT } \\
0-<4\end{array}$ & $\begin{array}{c}\text { PT } \\
4-15\end{array}$ & $\begin{array}{c}\text { GS } \\
0-<4\end{array}$ & $\begin{array}{c}\text { GS } \\
4-15\end{array}$ \\
\hline $\begin{array}{l}\text { Overall IOC for models as used in LYFT } \\
\text { score }^{1}\end{array}$ & See footnotes for changes in these scores & $0.66^{2}$ & $0.60^{3}$ & $0.67^{4}$ & $0.68^{5}$ & $0.59^{6}$ & $0.57^{7}$ \\
\hline Candidate angina noted & Low impact on LYFT score & 1.257 & 1.191 & 1.304 & 1.147 & 1.175 & 1.144 \\
\hline Candidate cerebrovascular disease missing & $\begin{array}{l}\text { Inconsistent results in various age- and } \\
\text { diabetes-specific models, low impact on } \\
\text { LYFT }\end{array}$ & 1.047 & 1.144 & 1.052 & 1.316 & 1.050 & 1.164 \\
\hline Candidate cerebrovascular disease & $\begin{array}{l}\text { Inconsistent results in various age- and } \\
\text { diabetes-specific models, low impact on } \\
\text { LYFT }\end{array}$ & 1.255 & 1.242 & 1.223 & 1.393 & 1.206 & 1.287 \\
\hline Candidate peripheral vascular disease & Low impact on LYFT score & 1.389 & 1.347 & 1.433 & 1.227 & 1.241 & 1.148 \\
\hline Candidate previous malignancy & $\begin{array}{l}\text { Inconsistent results in various age- and } \\
\text { diabetes-specific models, low impact on } \\
\text { LYFT }\end{array}$ & 1.191 & 1.156 & 1.182 & 1.105 & 1.109 & 1.004 \\
\hline Candidate previous malignancy missing & $\begin{array}{l}\text { Inconsistent results in various age- and } \\
\text { diabetes-specific models, low impact on } \\
\text { LYFT }\end{array}$ & 1.040 & 1.124 & 1.028 & 1.308 & 1.046 & 1.154 \\
\hline Candidate female (ref = male) & $\begin{array}{l}\text { Low LYFT impact, inappropriate for } \\
\text { allocation }\end{array}$ & 0.981 & 0.979 & 0.912 & 0.920 & 0.944 & 0.929 \\
\hline Candidate insurance: private primary & Low LYFT impact, not medical criterion & 0.887 & 0.942 & 0.797 & 0.710 & 0.803 & 0.728 \\
\hline Candidate insurance: other/missing & Low LYFT impact, not medical criterion & 1.021 & 1.105 & 0.979 & 1.160 & 0.978 & 1.025 \\
\hline Candidate insurance: public primary & Low LYFT impact, not medical criterion & (ref) & (ref) & (ref) & (ref) & (ref) & (ref) \\
\hline Candidate drug-treated hypertension & $\begin{array}{l}\text { Manipulatable, thus not appropriate for } \\
\text { allocation }\end{array}$ & 1.039 & 0.949 & 1.001 & 0.963 & 0.989 & 0.977 \\
\hline $\begin{array}{l}\text { Candidate drug-treated hypertension } \\
\text { missing }\end{array}$ & $\begin{array}{l}\text { Manipulatable, thus not appropriate for } \\
\text { allocation }\end{array}$ & 0.986 & 1.188 & 1.039 & 1.344 & 1.036 & 1.176 \\
\hline Candidate on peritoneal dialysis at listing & $\begin{array}{l}\text { Manipulatable, thus not appropriate for } \\
\text { allocation }\end{array}$ & 1.282 & 1.145 & 0.88 & 0.856 & 0.855 & 0.828 \\
\hline $\begin{array}{l}\text { Candidate dialysis modality at listing } \\
\text { missing or none }\end{array}$ & $\begin{array}{l}\text { Manipulatable, thus not appropriate for } \\
\text { allocation }\end{array}$ & 0.964 & 1.144 & 0.901 & 1.143 & 0.868 & 1.006 \\
\hline Candidate on hemodialysis at listing & $\begin{array}{l}\text { Manipulatable, thus not appropriate for } \\
\text { allocation }\end{array}$ & (ref) & (ref) & (ref) & (ref) & (ref) & (ref) \\
\hline Candidate race/ethnicity African American & $\begin{array}{l}\text { Not objective, thus not appropriate for } \\
\text { allocation }\end{array}$ & 0.713 & 0.809 & 1.006 & 1.145 & 1.338 & 1.441 \\
\hline Candidate race/ethnicity other or missing & $\begin{array}{l}\text { Not objective, thus not appropriate for } \\
\text { allocation }\end{array}$ & 0.600 & 0.767 & 0.758 & 0.668 & 0.777 & 0.786 \\
\hline Candidate race/ethnicity Hispanic & $\begin{array}{l}\text { Not objective, thus not appropriate for } \\
\text { allocation }\end{array}$ & 1.152 & 1.032 & 0.985 & 1.191 & 1.111 & 1.223 \\
\hline $\begin{array}{l}\text { Candidate race/ethnicity White } \\
\text { (non-Hispanic) }\end{array}$ & $\begin{array}{l}\text { Not objective, thus not appropriate for } \\
\text { allocation }\end{array}$ & (ref) & (ref) & (ref) & (ref) & (ref) & (ref) \\
\hline 0 A mismatch (MM) & Low LYFT impact, contributes to inequity & & & 0.957 & 0.975 & 0.898 & 0.953 \\
\hline $1 \mathrm{AMM}$ & Low LYFT impact, contributes to inequity & & & 0.942 & 0.993 & 0.924 & 0.987 \\
\hline $0 \mathrm{~B} \mathrm{MM}$ & Low LYFT impact, contributes to inequity & & & 0.942 & 0.964 & 0.885 & 0.919 \\
\hline $1 \mathrm{~B} \mathrm{MM}$ & Low LYFT impact, contributes to inequity & & & 0.954 & 0.986 & 0.930 & 0.952 \\
\hline Candidate diagnosis: glomerularnephritis ${ }^{8}$ & Low LYFT impact, not objective & (ref) & 1.155 & (ref) & 1.301 & (ref) & 1.534 \\
\hline Candidate diagnosis: hypertension & Low LYFT impact, not objective & 1.026 & 1.145 & 1.162 & 1.706 & 1.239 & 1.878 \\
\hline Candidate diagnosis: other/missing & Low LYFT impact, not objective & 1.230 & 1.174 & 1.103 & 1.757 & 1.065 & 1.739 \\
\hline
\end{tabular}

Bold italics indicate $\mathrm{P}<0.05$.

${ }^{1}$ Changes in IOC scores reported for each variable for each model reflect the change resulting in including the entire variable group (e.g. Candidate insurance: private primary, public primary and other/missing) into the model used for LYFT. The IOC was calculated by splitting the data used to make the models into two groups; one for model-building and one for testing. Changes that did not result in an altered IOC within the first two decimal places after rounding were not reported.

${ }^{2}$ Waiting list model for 0 to $<4$ years: IOC did not increase beyond 0.66 for any variable group in this table.

${ }^{3}$ Waiting list model for 4-15 years: IOC increased for candidate race (0.63).

${ }^{4}$ Posttransplant patient survival model for 0 to $<4$ years: IOC increased for insurance $(0.68)$.

${ }^{5}$ Posttransplant patient survival model for 4-15 years: IOC increased for angina (0.69), cerebrovascular disease (0.69), sex (0.69), insurance (0.69), race (0.69) and additional diagnosis categories (0.69).

${ }^{6}$ Posttransplant graft survival model for 0 to $<4$ years: IOC increased for insurance (0.60), dialysis modality $(0.60)$ and race $(0.60)$.

${ }^{7}$ Posttransplant graft survival model for $4-15$ years: IOC increased for race $(0.58)$.

${ }^{8}$ Because candidate diagnosis: polycystic is in the 0-4-year models, glomerularnephritis is the reference category for these models. In the 4- to 15-year models, polycystic diagnosis is the reference when the diagnosis variable group is included in the model. 
Verifying proportional hazards in LYFT covariates. Statistical tests were performed to determine whether there were any significant changes in the variable effects over time within each period ( 0 to $<4$ years vs. $4-15$ years on the waiting list or after transplant). Every main effect covariate used in the LYFT calculation was tested in each of the models to determine if the proportional hazard assumption held during all periods. Each test was done in a separate model, where the variable being tested was included both as a baseline characteristic and as a time-dependent characteristic interacting multiplicatively with linear time.

To speed processing, a one-tenth random sample of candidates and recipients was used. This left 2608 candidates with 685 events for the smallest data set; namely the 4to 15 -year period for survival without transplant. The other three models had between 1700 and 2400 events.

The covariates indicating diabetes and whether the recipient received an SPK transplant were known to be nonproportional before this testing. As a consequence, these tests were performed on models in which these factors were stratified into three groups: nondiabetic recipients, diabetic kidney recipients and diabetic kidney-pancreas recipients.

Of the 70 tests of time-dependent covariates conducted, four were statistically significant with p-values below 0.05 . The four factors were: zero HLA mismatch (MM) (posttransplant model for the 4- to 15-year period, parameter $=-0.13, p=0.05$ ), cohort year (without-transplant model for the 4- to15-year period, parameter $=0.015, p=0.04$ ), preemptive listing and still not on dialysis at offer (withouttransplant model for the 4- to 15-year period, parameter $=-0.081, p=0.04$ ), log of donor weight (posttransplant model for the 4- to 15-year period, parameter $=0.17, p=$ $0.01)$. These factors will be investigated further but were left as proportional hazards in this iteration of the LYFT modeling. This approach will account for these factors on the basis of their average effect during the follow-up interval.

The proportional hazards assumption seems to hold overall in each of the models used, and no time-dependent factors have been included in the LYFT calculations.

Kidney alone versus simultaneous kidney-pancreas, diabetic versus nondiabetic. The waiting list models for kidney nondiabetic, kidney diabetic and kidney-pancreas survival showed that the death rates for these three groups were close to proportional throughout 10 years of follow-up and that the kidney nondiabetic and kidney diabetic groups were nearly proportional for years 10-17. The models for survival with transplant for kidney nondiabetic, kidney diabetic and kidney-pancreas survival showed that the death rates for these three groups were not proportional.
Table 5: Factors not significantly predictive of survival $4-15$ years after offer or transplant

\begin{tabular}{|c|c|c|}
\hline $\begin{array}{l}\text { Survival without } \\
\text { transplant } \\
\text { (WL) }\end{array}$ & $\begin{array}{l}\text { Survival with } \\
\text { transplant } \\
\text { (PT) }\end{array}$ & $\begin{array}{l}\text { Graft } \\
\text { survival } \\
\text { (GS) }\end{array}$ \\
\hline Body mass index & Candidate albumin & Candidate albumin \\
\hline \multirow[t]{6}{*}{$\begin{array}{l}\text { Candidate peak } \\
\text { panel-reactive } \\
\text { antibody }\end{array}$} & $\begin{array}{l}1 \text { DR mismatch (MM) } \\
\text { (reference = 2 DR } \\
\text { MM) }\end{array}$ & $\begin{array}{l}\text { Donor age }(<18 \\
\text { years) }\end{array}$ \\
\hline & Donor age & $\begin{array}{l}\text { Donor cause of } \\
\text { death: anoxia }\end{array}$ \\
\hline & $\begin{array}{l}\text { Donor cause of } \\
\text { death: anoxia }\end{array}$ & $\begin{array}{l}\text { Donor cause of death: } \\
\text { central nervous } \\
\text { system tumor }\end{array}$ \\
\hline & $\begin{array}{l}\text { Donor cause of death: } \\
\text { central nervous } \\
\text { system tumor }\end{array}$ & Donor hypertension \\
\hline & $\begin{array}{l}\text { Donor } \\
\text { cytomegalovirus } \\
\text { negative }\end{array}$ & Donor weight \\
\hline & $\begin{array}{l}\text { Donor hypertension } \\
\text { Donor weight }\end{array}$ & Previous transplant \\
\hline
\end{tabular}

All Cox models of survival after transplant used to estimate LYFT are stratified into three groups: nondiabetic kidney transplants, diabetic kidney transplants and kidneypancreas transplants with different regression coefficients used in each group, when there was evidence that the coefficients differed by group. Unlike the models of survival after transplant, the model of candidate survival without transplant did not stratify by diabetes status or transplant type. This allowed the use of the proportional hazards assumption to complete the kidney-pancreas survival curve through 15 years, as there were insufficient kidney-pancreas candidates with survival beyond 10 years to define a separate survival curve. In other words, the hazards for kidney-pancreas candidates were assumed to continue to be proportional to those of kidney candidates after the period during which the hazard for kidney-pancreas candidates could be independently estimated. This assumption meant kidney-pancreas candidate survival without transplant could be estimated based on functions of kidney candidate survival without transplant.

Covariates used to estimate short-term and long-term survival. Some factors used in the LYFT models that were significant predictors of short-term ( 0 to $<4$ year) survival were not predictive of long-term survival and were thus not included in the long-term (4-15 year) models (Table 5). The use of separate models for short-term and long-term survival also allowed the effects of covariates to differ for short- versus long-term survival, thereby allowing for possible nonproportional effects.

\section{Testing interactions}

Both clinical judgment and statistical analysis were used to determine the interaction terms used in the LYFT calculations. Table 6 shows the interaction terms used. 
Table 6: Interaction terms used in LYFT calculations

Candidate age interaction with kidney (KI)-alone diabetes

Candidate age interaction with SPK

Candidate albumin interaction with simultaneous

kidney-pancreas transplant (SPK)

Candidate BMI interaction with SPK

PRA $10+$ interaction with SPK

Candidate previous transplant interaction with SPK

Candidate had not developed full end-stage renal disease

(ESRD) by sample date with Kl-alone diabetes

Candidate had not developed full ESRD by sample date with SPK

\section{Testing linearity of effect}

Candidate age at offer or transplant. Candidate age at offer or transplant was found to be a nearly linear effect, as shown in Figure 4, which displays the log-hazard ratio (i.e. the log of the relative death rate, compared with that of 18-year-old nondiabetics) versus candidate age, where the slope for candidate age is allowed to change at specified ages according to the data.

After reviewing the results shown in Figure 4 and similar results for transplant recipients, the effect of age on death rates in each of the regression models was included as a continuous, linear age predicting the log-hazard ratio. Interaction terms between age and diabetes (DM) for kidneyalone ( $\mathrm{KI}$ non-DM and $\mathrm{KI} \mathrm{DM}$ ) and kidney-pancreas (KP $\mathrm{DM})$ were included in the without-transplant model to account for differences in the effects of age between patients with and without diabetes. Interaction terms between age and diabetes were also included in the with-transplant model.

All other covariates. Several factors were found to have nonlinear effects on the outcome in at least one of the models. For certain terms (e.g. time on dialysis, donor weight) log transformations resulted in a better fit according to likelihood ratio tests. For panel-reactive antibody (PRA) values, categorical variables for different levels seemed the best approach to the OPTNKC, since the existing national kidney allocation policy treats highly sensitized (PRA $80 \%$ or

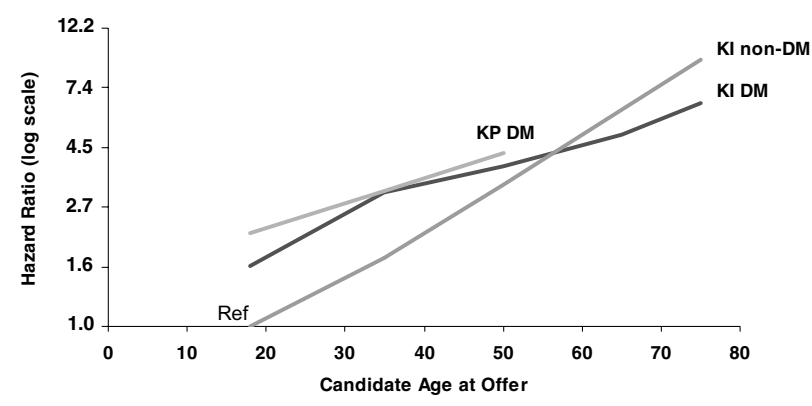

Source: SRTR analysis, May 2007.

Figure 4: Patterns of death risk (without transplant) by age for diabetics versus nondiabetics.
Table 7: Nonlinear parameterizations of continuous variables used in LYFT

\begin{tabular}{ll}
\hline Variable & Type of nonlinear effect \\
\hline $\begin{array}{l}\text { Candidate peak panel- } \\
\text { reactive antibody }\end{array}$ & Categories: 0-10, 11-79, \\
Donor weight in kg & $80-100$ \\
Years since start of dialysis & Log transformation (In[weight +1 ]) \\
Donor age & Slope change at 18 \\
Candidate body mass index & Slope change at 20 \\
Candidate albumin & Slope change at 3.5 \\
\hline
\end{tabular}

higher) candidates differently. Other nonlinear effects were modeled using splines. Instead of a single straight line, the spline approach models the effect as a series of connected straight lines, where the slope was allowed to change as dictated by the data (as illustrated in Figure 4).

If a nonlinear effect was found for one model, it was kept for all models that included that covariate. Table 7 lists the factors where nonlinear terms were used.

\section{Long-Term Survival and Extrapolation}

\section{Long-term survival based on older data}

Older data were used to obtain the long-term survival estimates, but survival levels were adjusted to reflect recent experience. The survival curves for each cohort year shown in Figure 5 illustrate this process. The shape of the survival curve for the year 2006 nondiabetic kidney transplant recipients (topmost) is solid for 1 year, where actual data are available, and dotted afterward, indicating the extrapolation period. The extrapolation of this curve is based on older experience, as shown by the solid lines for previous years; but, the fact that 2006 recipients have had better survival than their counterparts in previous cohorts during the comparable period after transplant is also accounted for. The baseline survival curve in each LYFT calculation is estimated out to year 15 using a Cox model with indicator variables for each cohort year with adjustment, in a manner similar to the example adjustment for the year 2006 shown in Figure 5.

Amount of extrapolation required. When calculating the median remaining lifespan for candidates active on the waiting list on a given date, (e.g. 1 January 2004), only about $1 \%$ require extrapolation beyond the 15 -year survival curve described in the prior section. Transplant recipients have longer lifespans, and about $28 \%$ of the candidates active on the list on 1 January 2004 would require extrapolation to determine their median lifespan if they were to receive a non-ECD kidney.

For purposes of illustration, the following histograms (Figure 6 and Figure 7) show the distributions of remaining lifespans for adult candidates active on the kidney (or 
Wolfe et al.

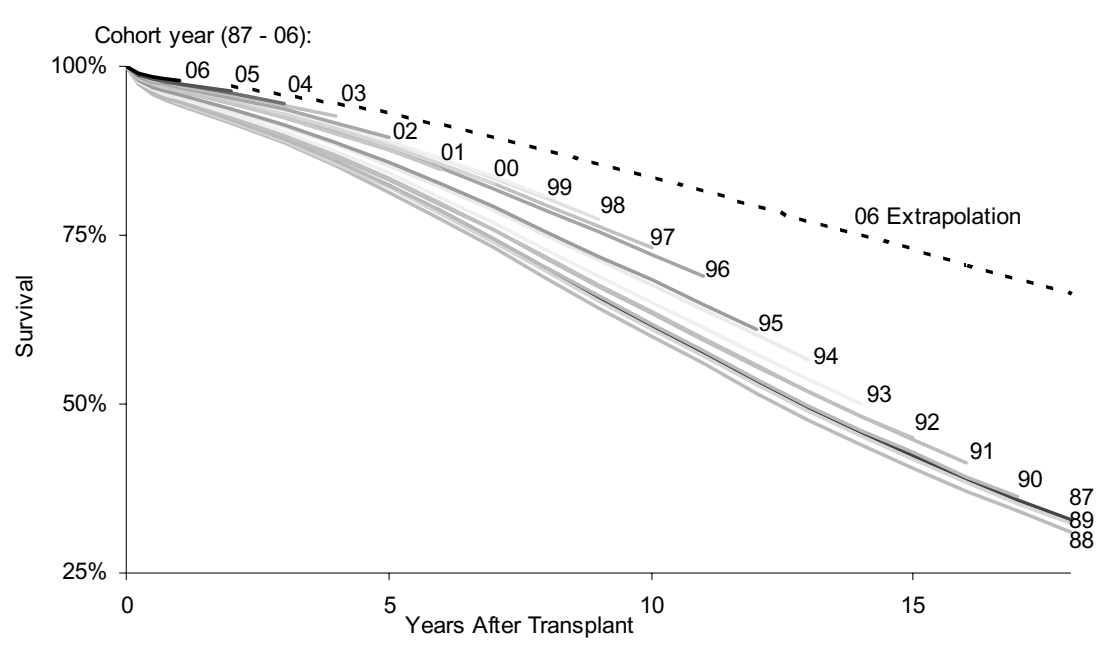

Source: SRTR analysis, May 2007

kidney-pancreas) waiting list on 1 January 2004, if they never received a transplant (Figure 6) and if they all received a non-ECD kidney (or kidney-pancreas) from a donor with the mode characteristics of actual donors from the 2003 deceased donor pool (Figure 7).

\section{Details of extrapolation beyond 15 years of median sur-} vival. While the initial hazard after transplant is elevated, the log of the long-term (more than 4 years) hazard appears to be reasonably well approximated as linear growth over time, suggesting that the growth in death rates can be approximated as exponential growth with time (7). The linear growth in the log hazard over time is also similar (proportional) to that of the general population, as shown by the roughly parallel plots of the death rate per year on the log scale in Figure 8. Upper curves in this figure indicate the death rate per year (starting 4 years after offer or transplant) for otherwise-average candidates who at age 45 years either remain untransplanted (uppermost line, starting 4 years after an arbitrary offer), receive a kidney transplant as a diabetic (second highest line), receive a SPK

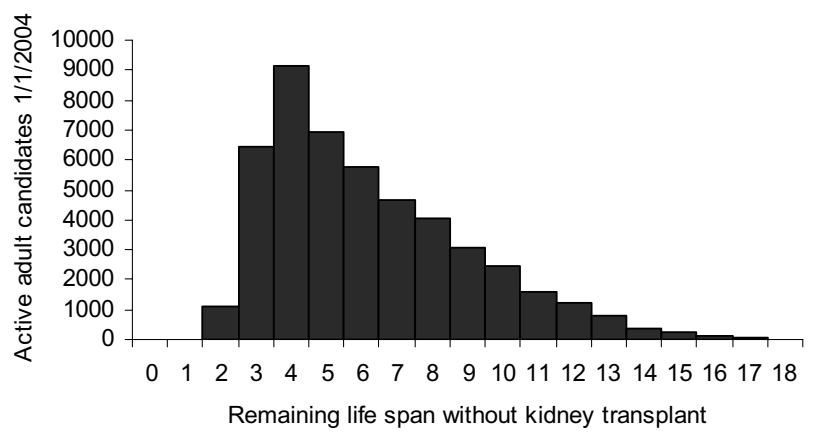

Source: SRTR analysis, August 2007

Figure 6: Distribution of estimated median lifespans without transplant for active (as of 1 January 2004 ) adult candidates.
Figure 5: Patterns of mortality by cohort year of transplant. transplant (third highest line) or receive a kidney transplant as a nondiabetic (fourth line). The death rates start 4 years after offer or transplant for each group in order to emphasize the trend associated with increasing age, rather than the relatively brief postoperative elevation in death rates in the transplant groups. The slopes used for extrapolation in the LYFT calculation, obtained through linear regression using all patients, are detailed in Table 8. The absolute mortality remains lowest for the general population and highest for the waiting list. Posttransplant mortality increases with time in a manner similar to that of the general population, while waiting list mortality, although higher overall, may increase more slowly with time.

\section{LYFT Scores Versus Candidate Demographics}

The histogram in Figure 9 shows the overall distribution of LYFT scores for adult kidney and kidney-pancreas candidates active on the waiting list on 1 January 2004 had they each received an average non-ECD kidney.

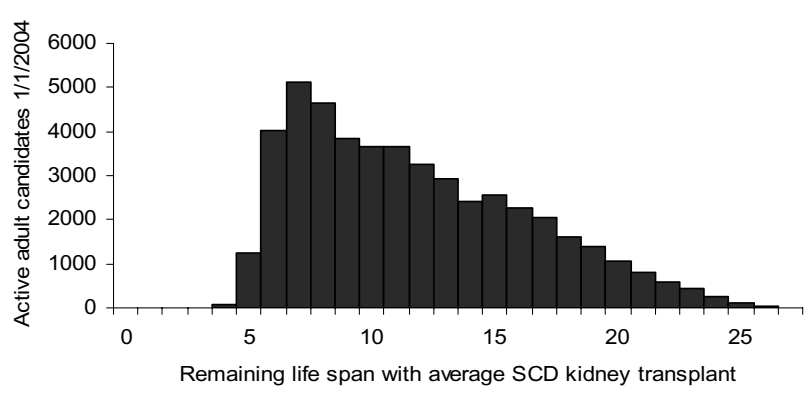

Source: SRTR analysis, August 2007

Figure 7: Distribution of estimated median lifespans with transplant for active (as of 1 January 2004 ) adult candidates. 


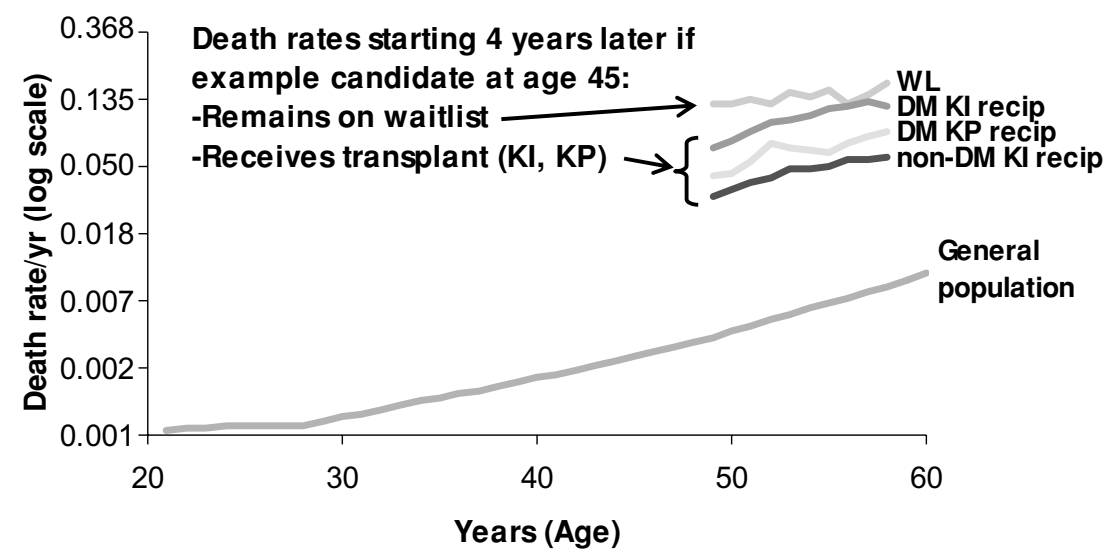

Figure 8: Comparison in increase in death rate over time among general population and candidates with and without transplant.

\author{
General population death rates are by age, WL and PT death rates \\ are for average 45-year-old from 4 years after offer/transplant \\ Source: SRTR analysis, July 2006
}

\section{Box plots of distribution of LYFT by demographic}

Each box plot (Figures 10-16) shows the distribution of LYFT scores among kidney and kidney-pancreas candidates active on the waiting list on 1 January 2004 using the box for the interquartile range (25th through 75th percentile) and whiskers for the 5th and 95th percentiles. The horizontal line within the box is the median LYFT score for the population depicted. The LYFT scores are calculated based on a kidney or kidney-pancreas donor with the average characteristics of a non-ECD donor; averages apply to both donor-alone factors such as donor age (32 years) and to shared donor/recipient factors such as level of HLA mismatch. The distribution of candidate variables (e.g. body mass index [BMI], diagnosis, time since starting dialysis, etc.) reflects those actually seen in the candidate and recipient populations in 2003, the year represented in the model. The distribution of LYFT scores within the box and whisker plots reflects the effects of these variables among the represented patients.

Figure 10 demonstrates the range and median of LYFT scores for all candidates by age and diagnosis categories. The percentages at the top of the figure represent the proportion of these individuals in the candidate population. LYFT scores in general are higher among younger candi-

Table 8: Slope of long-term log-hazard over time

Survival without transplant (waiting list [WL]): 0.044

Survival after transplant for nondiabetic kidney recipient (KI DM): 0.081

Survival after transplant for diabetic kidney recipient (KI DM): 0.090

Survival after transplant for kidney-pancreas recipient (KP DM): 0.081

Graft survival for nondiabetic kidney recipient (KI DM): 0.036

Graft survival for diabetic kidney recipient (KI DM): 0.071

Graft survival for kidney-pancreas recipient (KP DM): 0.038 dates and for diabetic candidates awaiting an SPK transplant; they are lower for older candidates and for diabetic candidates listed for kidney transplantation alone. The distributions of the LYFT scores are very similar across categories of other patient subpopulations (diagnosis among nondiabetics, race/ethnicity, sex, insurance status, blood type and DSA) as shown in Figures 11-16.

\section{Independent effects on LYFT (all else equal)}

Figures 17-20 demonstrate the estimated LYFT for a hypothetical group of almost-identical candidates who only differ by the factor noted. In contrast to the box plots (Figures 10-16) that display the range of LYFT scores among actual candidate groups (which tend to differ by more than one factor), these graphics show the estimated relative effects of certain example factors on LYFT. The purpose of Figures 17-20 is to show the effect of single-example factors on LYFT with all else held equal, not to depict ranges of LYFT scores for actual candidate groups.

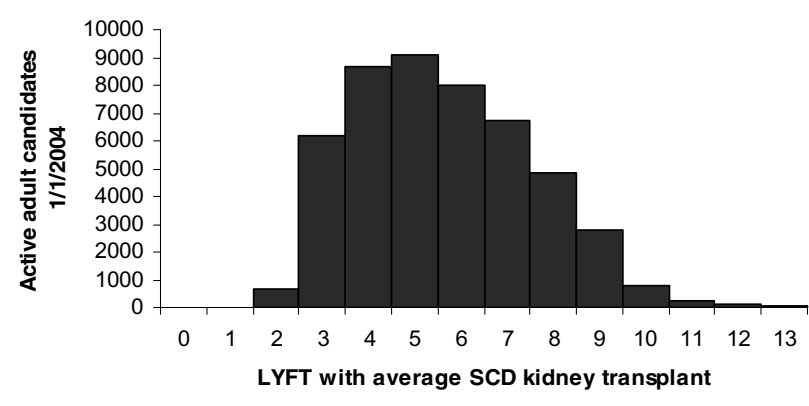

Source: SRTR analysis, August 2007

Figure 9: Distribution of LYFT among active (as of 1 January 2004) adult candidate lifespans with transplant. 
Wolfe et al.

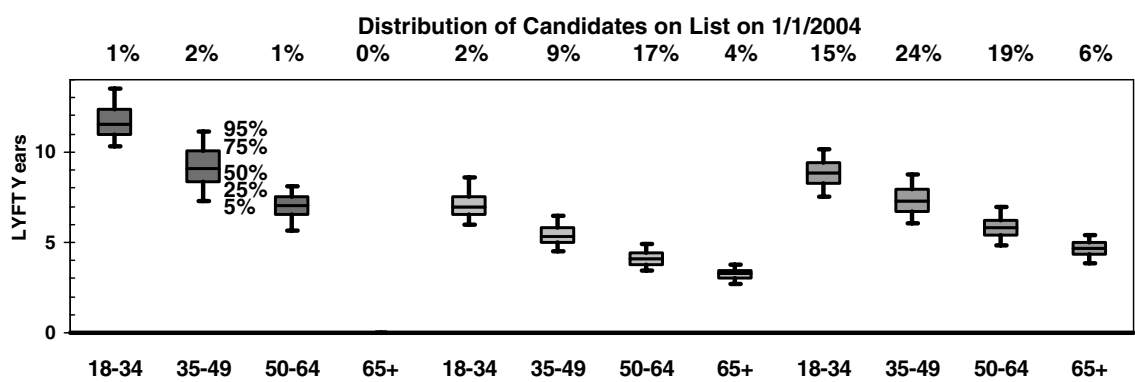

\section{Diabetic (KP) Diabetic (KI) Non-Diabetic (KI)}

Source: SRTR analysis, August 2007.

Figure 10: Distribution of LYFT by candidate age (years) and diabetes status.

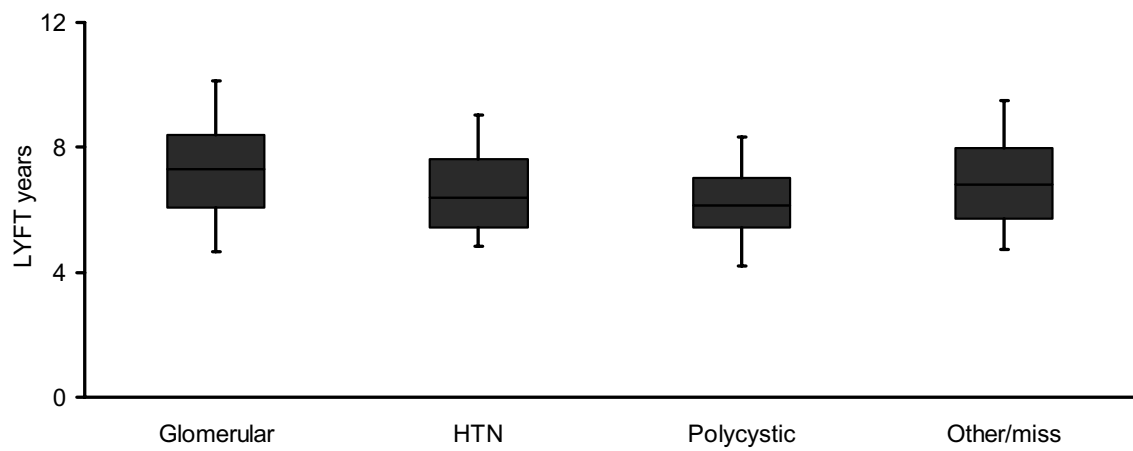

Source: SRTR analysis, August 2007.

Figure 11: Distribution of LYFT by nondiabetic diagnosis.

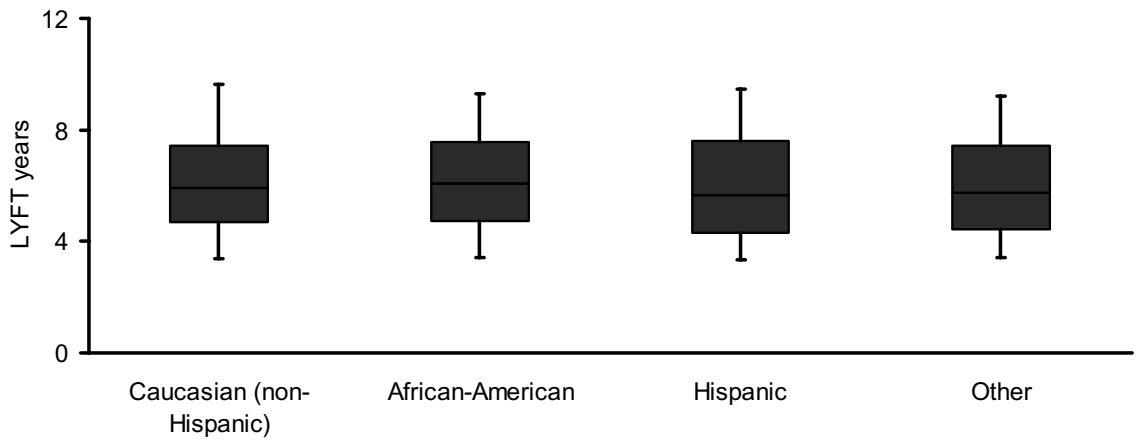

Source: SRTR analysis, August 2007.

Figure 12: Distribution of LYFT by candidate race/ethnicity.

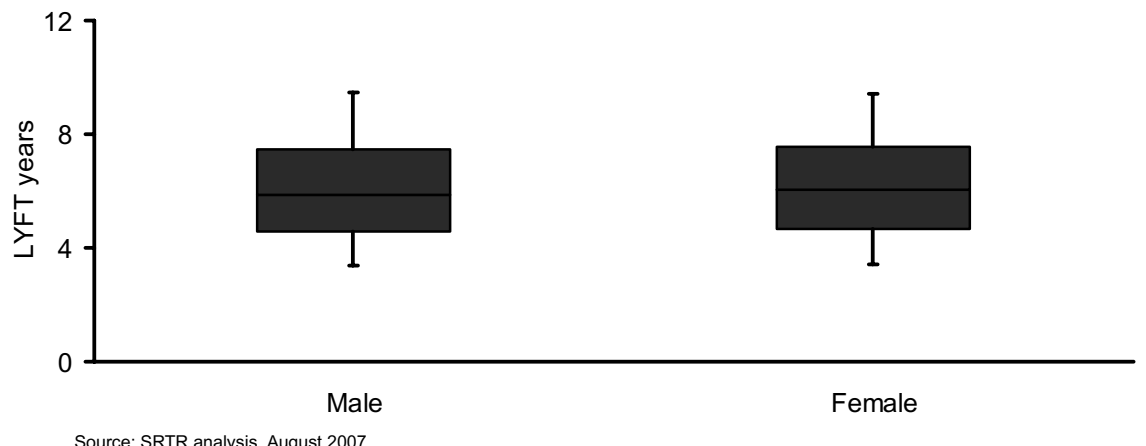

Figure 13: Distribution of LYFT by candidate sex. 
Figure 14: Distribution of LYFT by candidate primary insurance status.

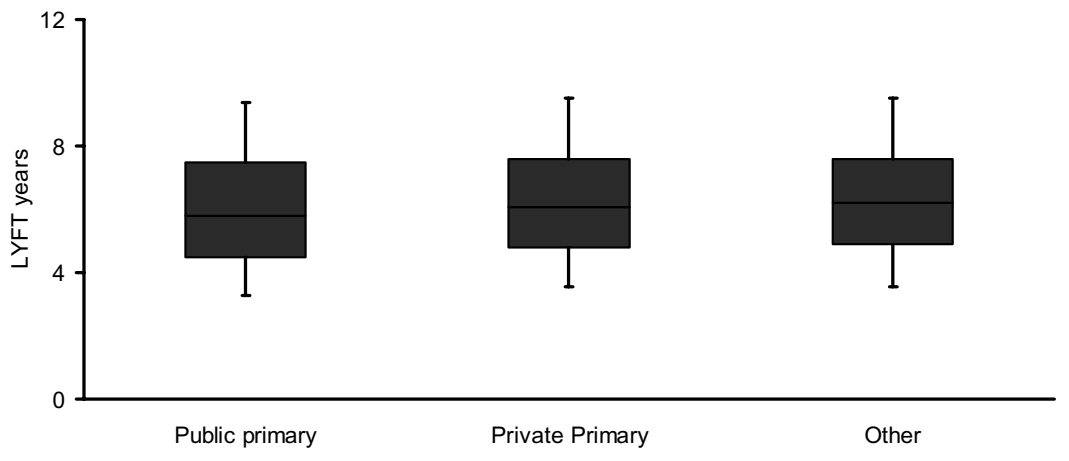

Public $=$ Medicare or Medicaid as primary or sole insurer. Private $=$ Private insurance as primary or sole insurer Other $=$ Other, no insurance, or missing data

Source: SRTR analysis, August 2007.

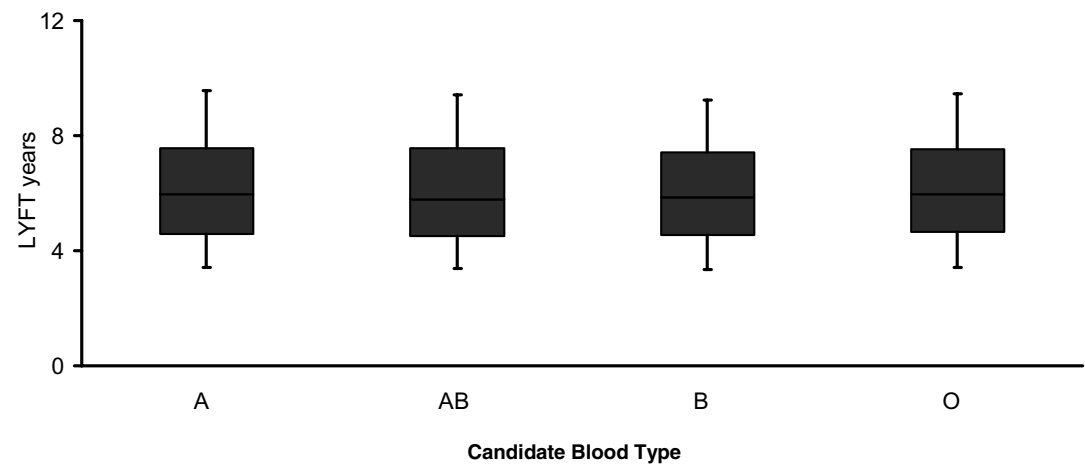

Source: SRTR analysis, August 2007

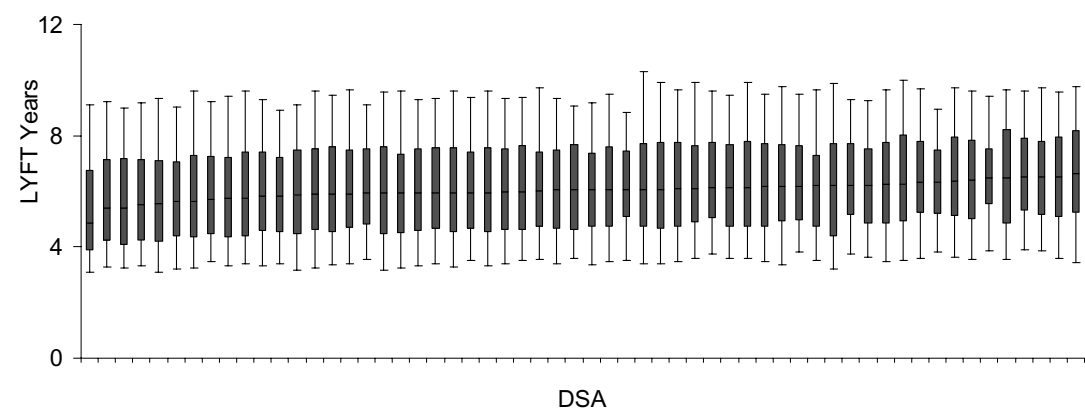

Source: SRTR analysis, August 2007

didate donation service area (DSA).

\section{Discussion}

LYFT, estimated using regression models based on candidate and donor data, provides information about the quality-adjusted extra years of life that a given transplant could be expected to provide for a given patient. This could be a valuable tool in allocation of deceased-donor kidneys, evaluation of kidney allocation methods and patient counseling. Prioritizing the candidates with the higher LYFT scores for each kidney that becomes available could increase the average extra years of life of a transplant by about 1 year compared with the current system; accordingly, a year's worth of kidneys allocated in this way could provide in aggregate around 10000 extra years of life (8).

The concept of LYFT is useful both for designing an effective organ allocation system and for advising candidates of treatment options.

1. In organ allocation, LYFT could be calculated when a kidney becomes available, based on the donors characteristics and those of the candidates active on the waiting list. Prioritizing offers to candidates with large LYFT scores would lead to more years of life among candidates and recipients, in total. 


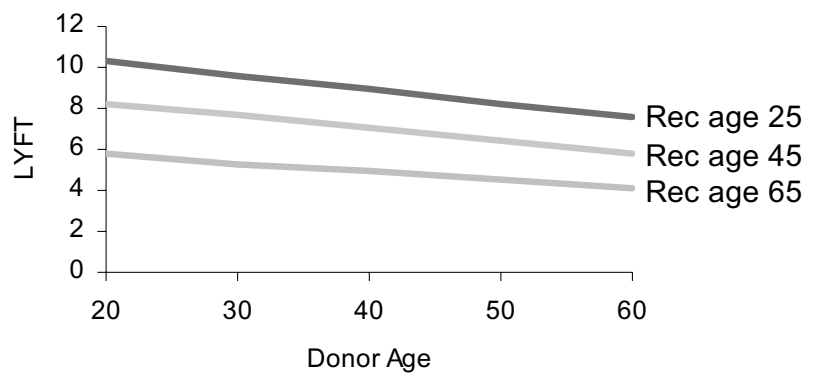

Source: SRTR analysis, August 2007

Figure 17: Donor and candidate age in years versus LYFT.

2. When informing a single candidate of personal treatment options, LYFT could be calculated using the characteristics of that candidate for a range of several possible donor kidneys that might become available in the future (e.g. ECD, non-ECD and living donor). This information, along with candidate health status and the likelihood of receiving various types of kidneys, could be used, for example, to make informed decisions about whether to rule out offers of certain types of donor kidneys for a specific candidate.

A kidney allocation system incorporating LYFT could be modified to incorporate goals other than that of increasing the total candidate lifespan. For example, the LYFT scores shown in this article, reflecting the direction chosen by the OPTNKC, are modified by a conversion factor for quality of life. Years with a functioning transplant are given a weight of one, whereas waiting list years and years after graft failure are given a weight of 0.8 . Calculation of the expected years of graft survival allows the components of LYFT to be weighted according to these differences in quality of life. Other modifications to LYFT could include a weighting system that would discount years in the future compared with years occurring sooner or could add more emphasis to waiting list urgency. The models used to calculate LYFT are expected to be updated as additional data become available and refined as needed. Among the alternatives under consideration are the inclu-

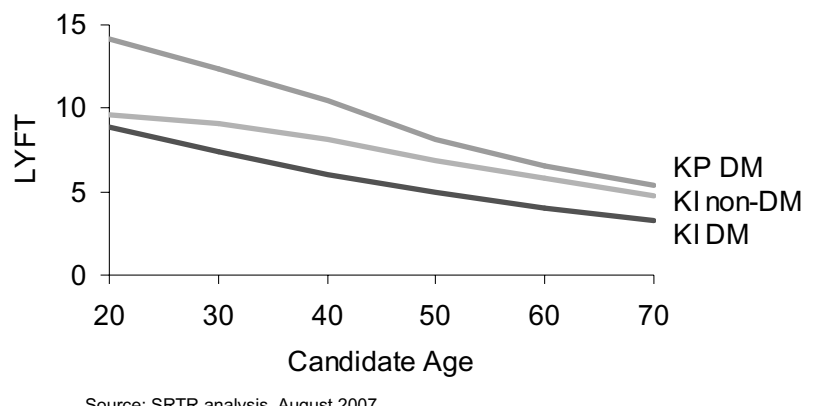

Figure 18: Candidate age and diabetes (kidney-pancreas and kidney-alone) versus LYFT.

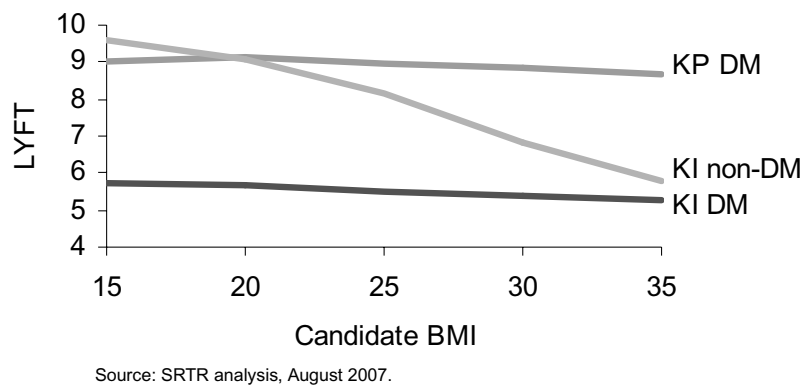

Figure 19: Candidate BMI and diabetes versus LYFT.

sion of ECD kidneys and the exclusion of SPK recipients in the models used to estimate LYFT. In addition, other elements could be blended into the kidney allocation algorithm that would give allocation priority based upon considerations other than expected LYFT. Such factors as waiting time, PRA and prior donation serve other legitimate allocation priorities. Changes in LYFT resulting from modification of allocation rules can be used as a metric to gauge the consequences of these rules on posttransplant patient survival.

\section{Summary}

LYFT, estimated using regression models based on candidate and donor data, provides information about the qualityadjusted survival benefit that a given transplant could provide a given patient. This measure can be estimated by Cox proportional hazards regression models without violating the assumptions inherent in using these models; it can incorporate a factor to account for the difference in quality of life with and without a functioning graft; LYFT can be estimated without extrapolating beyond available data for the majority of the candidates for a particular kidney; and it can be estimated using reasonable extrapolation methods for the remaining candidates. This could be a valuable tool in the allocation of deceased-donor kidneys, evaluation of kidney allocation methods and patient counseling. By prioritizing the candidates with the higher LYFT scores for each kidney that becomes available, each year's

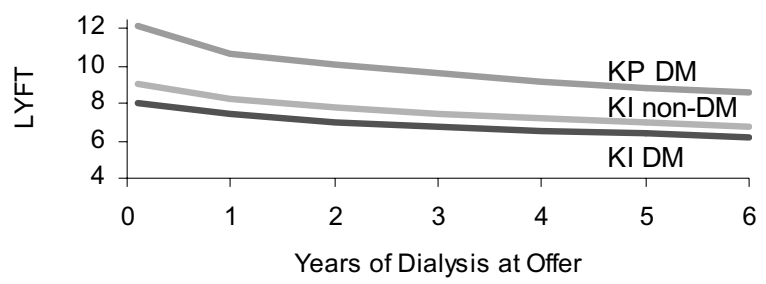

For preemptive candidates,

KP DM: 9.3

KI non-DM: 7.1

KI DM: 4.7

Source: SRTR analysis, August 2007

Figure 20: Candidate years on dialysis versus LYFT. 
worth of kidneys could provide over 10000 extra years of life beyond the benefit provided by the same kidneys under the current system (7).

\section{Acknowledgment}

The Scientific Registry of Transplant Recipients is funded by contract number 234-2005-37009C from the Health Resources and Services Administration (HRSA), US Department of Health and Human Services. The views expressed herein are those of the authors and not necessarily those of the US Government. This is a US Government-sponsored work. There are no restrictions on its use. This article was produced as part of the 2007 OPTN/SRTR Annual Report. The Annual Report gathers information on many aspects of solid organ transplantation in one publication. More information can be found at www.ustransplant.org.

\section{References}

1. McCullough KP, Leichtman AB, Port FK, Wolfe RA. Trends in kidney recipient age and survival benefit due to transplant by year. Am J Transplant 2007; 7: 231 (abstract).
2. Wolfe RA, Ashby VB, Milford EL et al. Comparison of mortality in all patients on dialysis, patients on dialysis awaiting transplantation, and recipients of a first cadaveric transplant. N Engl J Med 1999; 341: 1725-1730.

3. Marubini E, Valsecchi MG. 1995. Analysing Survival Data from Clinical Trials and Observational Studies. John Wiley \& Sons.

4. Andreas L, Keown P, Pus $N$ et al. A study of the quality of life and low cost-utility of renal transplantation. Kidney Int 1996; 50: 235-242.

5. Hornberger JC, Best JH, Garrison LP. Cost-effectiveness of repeat medical procedures: Kidney transplantation as an example. Med Decis Making 1997; 17: 363-372.

6. Wolfe RA. Avoiding statistical bias in predicting the life years from transplant (LYFT). p. 18. SRTR Working Paper. Available at: www.ustransplant.org. Accessed December 14, 2007.

7. Wolfe RA. Extrapolation methods: Approximating the median survival time beyond the duration of follow-up based on geometric and linear growth in death rates. SRTR Working Paper. Available at: www.ustransplant.org. Accessed December 14, 2007.

8. Wolfe RA, McCullough KP, Rodgers A, Leichtman AB. A modification to kidney transplant allocation to save more patient years of life. Am J Transplant 2007; 7: 230 (abstract). 